\title{
SEARCH FOR EXOTIC HADRONIC MATTER: TETRAQUARKS, PENTAQUARKS, DIBARYONS AND MESIC NUCLEI*
}

\author{
P. MOSKAL \\ The Marian Smoluchowski Institute of Physics, Jagiellonian University \\ Łojasiewicza 11, 30-348 Kraków, Poland
}

(Received December 3, 2015)

We describe selected results of the search for the exotic hadronic matter such as e.g. tetraquarks, pentaquarks and hexaquarks. The content of the proceedings is by far not complete and reflects a personal choice of the author with the emphasis put on the recent discovery of the dibaryon state and the search for the mesic nuclei with the WASA detector at COSY.

DOI:10.5506/APhysPolB.47.97

\section{Introduction}

In recent years, nearly half a century after the origin of the quark model $[1,2]$, a plethora of new hadrons was observed which cannot be classified as a meson (quark-anti-quark) or a baryon (three-quark) states. In this article, we will not go into the details of the nature of these newly discovered states because it is not yet established and it is still a topic of hot investigations. Instead, one of the aims of this proceedings is to try to make a link between two seemingly different fields of investigations: the search for new hadrons carried out at many high energy physics facilities, and a search for the mesic nuclei and dibaryons conducted at low energy hadron physics laboratories. The status of the investigations of tetraquarks (genuine $q q \bar{q} \bar{q}$ or meson-meson molecular-like states) and pentaquarks (genuine $q q q q \bar{q}$ or baryon-meson molecular-like state) is covered in the recent review [3]. Therefore, in this proceedings, as an introduction, we will only briefly report on a status of the search for the tetraquark and pentaquark, making a background to the next sections where more detailed account of the search for the mesic nuclei and the recent discovery of the dibaryon will be given.

\footnotetext{
* Presented at the LV Cracow School of Theoretical Physics "Particles and Resonances
} of the Standard Model and Beyond", Zakopane, Poland, June 20-28, 2015. 
Since decades, there have been known states as e.g. light scalars $\sigma$, $f_{0}(980)$ and $a_{0}(980)$ which include significant tetraquark contribution $[2,4,5]$. There are also suggestions that the light mass $1^{-+}\left(J^{P C}\right)$ exotics observed at BNL and CERN [6] may be dynamically generated (with strong tetraquark component) in $\eta^{\prime}-\pi$ re-scattering [7]. However, only in 2003, the first serious candidate for the heavy tetraquark state was discovered by the Belle Collaboration [8] as a narrow charmonium-like maximum (with the width less than $1.2 \mathrm{MeV}$ ) in the invariant mass of the $J / \psi \pi^{+} \pi^{-}$system produced in the decay of $B \rightarrow K J / \psi \pi^{+} \pi^{-}$. This result was confirmed by BaBar [9], LHCb [10] in the same and other [11, 12] decay channels of the $B$ meson and by CDF $[13,14]$ and D0 [15] in the $J / \psi \pi^{+} \pi^{-}$system produced in the $p \bar{p}$ collisions and by the LHCb and CMS in $p p$ collisions [16, 17], and by BES III in $e^{+} e^{-}$interaction [18]. The resonance is referred to as $X(3872)$. Its charge conjugation is positive since it decays to $\gamma J / \psi[9,19]$ and other quantum numbers $1^{++}\left(J^{P C}\right)$ were established based on the fivedimensional angular correlations between particles in $B^{+} \rightarrow X(3872) K^{+}$, $X \rightarrow \pi+\pi+J / \psi$ and $J / \psi \rightarrow \mu^{+} \mu^{-}$decay chains [10]. The $1^{++}$quantum numbers and its mass disfavor conventional states and suggest that the state is either a $D^{* 0}$ anti- $D^{0}$ molecule [20], genuine tetraquark [21] or a mixture of these states [22].

Recently, in 2015, the LHCb Collaboration reported discovery of a narrow resonance in the $J / \psi p$ channel with the mass of about $4450 \mathrm{MeV}$ and width of about $40 \mathrm{MeV}$, which is interpreted as a charmonium pentaquark [23] as was predicted by Karliner and Rosner [24]. However, so far, there is no common acceptance for the strange pentaquark, though one can find resonances of strange meson and nucleon as e.g. $N^{*}(1535)$, $\Lambda(1405)$ or $Z^{*}$, and though there are arguments in favor of taking some of these resonances seriously [25-30]. Some claims of the pentaquark observation were reported by LEPS [31] as a maximum in the missing mass spectrum of the $\gamma n \rightarrow K^{-} X$, and by DIANA [32] in the $K^{+} n \rightarrow p K^{0}$ process (such a resonance should have a uudds quark content). These were, however, not confirmed by the experiments in JLab [33] and most recent experiments at J-PARC [34-36], where the pentaquark $\Theta^{+}$was searched for in the $\pi^{-} p \rightarrow K^{-} X$ reaction applying a missing mass method. The research is continued and recently, the DIANA Collaboration reported new results with an evidence of the $\Theta^{+}$in the $K^{+} n \rightarrow K^{0} p$ process [37] with the width of $\Gamma\left(\Theta^{+}\right)=0.34 \pm 0.10 \mathrm{MeV}$ which is not excluded by the upper limits set by experiments at J-PARC [36].

The above examples illustrate that in recent years, an intensive search for exotic states changes rapidly the situation in the field of hadron physics revealing serious candidates built, at least to some extent, from tetraquark and pentaquark states. In the next section, we will report on the discovery of the dibaryon by the WASA-at-COSY Collaboration [38, 39], a state which may comprise contribution from the hexaquark. 


\section{Discovery of the dibaryon $d^{*}(2380)$}

The existence of the non-strange dibaryons (different from the deuteron) was predicted by Dyson and Xuong in 1964 [40], based on SU(6) symmetry of strong interactions. In the note added in proof, taking into account experimental results on $\pi^{+} d \rightarrow p p$ reaction [41], Dyson and Xuong predicted that the mass of the dibaryon $D_{03}$ (isospin $=0$, spin $=3$ ) should be equal to $2350 \mathrm{MeV}$. Though intensive experimental investigations have been carried out since then, only recently, after about half a century, a predicted $D_{03}$ dibaryon was discovered by the WASA-at-COSY Collaboration in the deuteron fusion associated with the production of the two neutral pions via $p n \rightarrow d \pi^{0} \pi^{0}$ reaction $[38,42]$. The observed signal is shown in the left panel of Fig. 1. The resonance has a maximum at the mass of about $2382 \mathrm{MeV}$ and the width of about $70 \mathrm{MeV}$. Its quantum numbers $I\left(J^{P}\right)=0\left(3^{+}\right)$were deduced based on the isospin of involved particles and angular distribution of the deuteron [38]. The observed structure is assigned to the dibaryon since it cannot be explained neither by the $N N^{*}(1440)$ production (dotted line) nor by the conventional $t$-channel $\Delta \Delta$ production (dashed line). As indicated by the dashed line in Fig. 1, in the case of $\Delta \Delta$ production, the excitation function should have maximum at about $80 \mathrm{MeV}$, higher mass and much larger width amounting to about $240 \mathrm{MeV}$. There are no baryonic resonances other than Roper $N^{*}(1440)$ and $\Delta \Delta$ which could explain the observed resonant shape of two-pion production in this energy range.
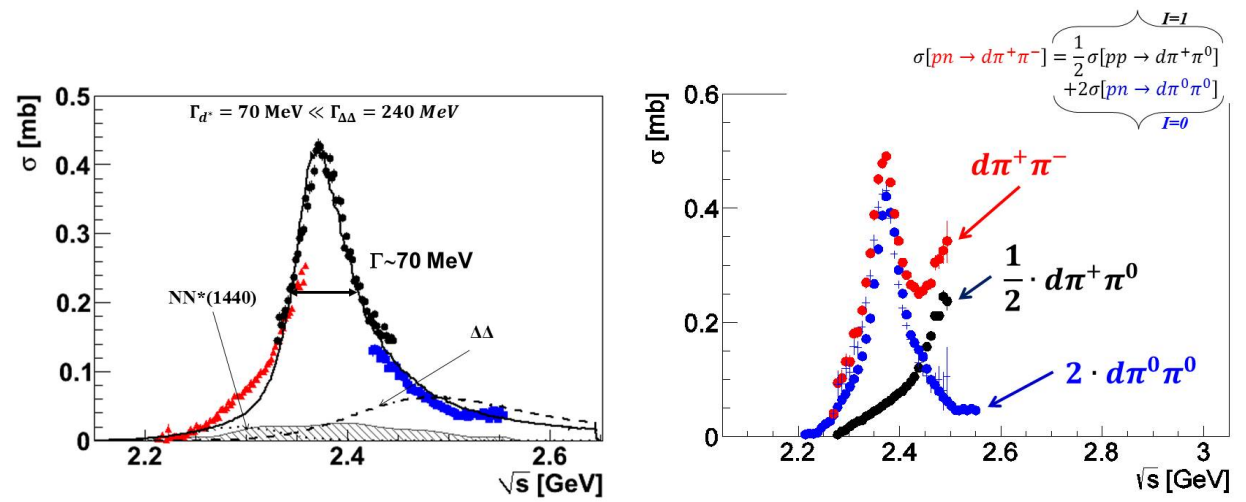

Fig. 1. (Left) Excitation function for the $p n \rightarrow d \pi^{0} \pi^{0}$ reaction determined by the WASA-at-COSY Collaboration [38]. (Right) Comparison between excitation functions determined by the WASA-at-COSY for the reactions $p n \rightarrow d \pi^{0} \pi^{0}, p n \rightarrow$ $d \pi^{+} \pi^{-}$and $p p \rightarrow d \pi^{+} \pi^{0}$ [43]. The figures are adapted from articles [38, 43] and from the conference talk of Bashkanov [44]. 
The hypothesis that the observed structure is a signal of the dibaryon is confirmed in the other deuteron fusion reactions where the signal from the dibaryon is observed as expected based on the isospin dependence [43]. The result of the comparison of the excitation functions for the reactions: $p n \rightarrow d \pi^{0} \pi^{0}, p n \rightarrow d \pi^{+} \pi^{-}$and $p p \rightarrow d \pi^{+} \pi^{0}$ is presented in the right panel of Fig. 1.

The resonance structure is also seen in agreement with predictions $[45,46]$ in the non-fusion reactions such as: $p n \rightarrow p n \pi^{0} \pi^{0}$ [47] and $p n \rightarrow p p \pi^{-} \pi^{0}$ [48].

Most importantly, the dibaryon is seen also in the proton-neutron elastic scattering. It manifests itself as a structure in the energy dependence of the analysing power (left panel of Fig. 2) and as a pole in the ${ }^{3} D_{3}$ partial wave, as seen in the Argand plot obtained from the partial wave analysis $[39,49]$. The above described observations undoubtedly reveal an existence of the dibaryon resonance $d^{*}(2380)$ which due to its quantum numbers is also referred to as $D_{03}$. Interestingly, the dibaryon $D_{03}$ survives in the nuclear medium and is observed also in the excitation function of the double pionic fusion of ${ }^{3} \mathrm{He}$ and ${ }^{4} \mathrm{He}$ via reactions $p d \rightarrow{ }^{3} \mathrm{He} \pi^{0} \pi^{0}[50,51]$ and $d d \rightarrow{ }^{4} \mathrm{He} \pi^{0} \pi^{0}$ [52]. As expected, the structure observed in the nuclear medium is broader with respect to the $D_{03}$ state produced in vacuum.
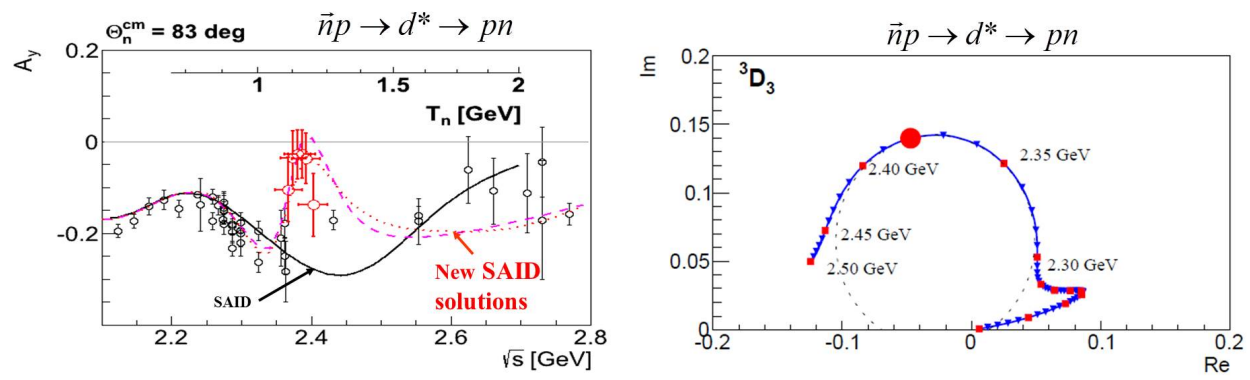

Fig. 2. (Left) Energy dependence of the beam analysing power for the $\vec{n} p \rightarrow n p$ scattering as determined by the WASA-at-COSY Collaboration [39, 49] (large open circles with horizontal bars) and previous experiments [53] (small open circles). (Right) Argand diagram for the ${ }^{3} D_{3}$ partial wave resulting from the partial wave analysis of the world data set extended by the new WASA-at-COSY result shown in the left panel. The analysis was performed by the SAID Data Analysis Center $[39,49]$. The figures are adapted from articles [39, 49] and from the conference talk of Bashkanov [44].

Based on the so far collected data, it was possible to establish that in $88 \%$ the $D_{03}$ dibaryon decays to $\Delta \Delta$ and only in $12 \%$ to proton-neutron $[44,54,55]$. Regarding its structure, the result of phenomenological analysis shows that it consists of $67 \%$ of hexaquark, $31 \%$ of S-wave $\Delta \Delta$ and $2 \%$ 
of D-wave $\Delta \Delta[44,56]$. There are also new approaches being developed which describe properties of the observed dibaryon, e.g. in the framework of the quark model [57] or in hadronic model with $N \Delta$ and $\Delta \Delta$ pion-assisted dibaryons [58-60] and extend the predictions to the dibaryons with strangeness [61] and charm [62].

\section{3. $\eta$-mesic helium}

The status of the search for the mesic nucleus was described in the recent reviews [63, 64], therefore here, we only briefly report on main results emphasizing perspectives of the search based on the high statistics data collected recently by the WASA-at-COSY Collaboration on proton-deuteron and deuteron-deuteron fusions near the threshold for the production of the $\eta$ meson.

A mesic nucleus is the system of the meson and nucleon bound by the strong interactions. It was predicted almost 30 years ago by Haider and Liu [65]. However, despite intensive theoretical [63] and experimental investigations of reactions induced by pions [66], protons [67], deuterons [68-73] and photons [74, 75], its existence was not confirmed so far.

The experimental studies are now concentrated on the search for the bound state of the nucleus with the meson which is electrically neutral as e.g. $\eta$ and $\eta^{\prime}$ [76-82], since in this case, the possible mesic system cannot be formed by the electromagnetic interaction and hence it can be bound only by the strong forces.

There are many indications pointing out that the $\eta$ meson is the best candidate for the creation of the mesic nucleus. For example, the interaction of the $\eta$ meson with nucleons is stronger compared to other possible candidates as e.g. pion or $\eta^{\prime}$ mesons, as it was inferred comparing the excitation functions for the production of these mesons in the proton-proton interaction [83-86] and by the comparison of the transparency ratio [87-89]. Another strong indication is a steep rise of the cross section at the threshold for the $\eta$ meson production in $p d \rightarrow^{3} \mathrm{He} \eta$ [90-93] and $d d \rightarrow^{4} \mathrm{He} \eta$ [94] reactions. The result for the $p d \rightarrow^{3} \mathrm{He} \eta$ process is shown in the right panel of Fig. 3. Though the $\eta^{\prime}$-nucleon interaction is weaker, there are also some indications in favor of the existence of the $\eta^{\prime}$-mesic nuclei, for example, the substantial mass reduction of the $\eta^{\prime}$ meson in medium associated with the axial U(1) symmetry in low energy QCD [95-98], the small width of the $\eta^{\prime}$ meson in nuclei deduced from the transparency ratio [88], and the observation that the real part of the $\eta^{\prime}$-nucleus potential is larger than its imaginary part [88, 99].

The studies performed so far reveal no significant signals and none of the claimed discoveries of the mesic nucleus is at present commonly accepted. In the case of the production of the $\eta$-mesic helium, the established upper limits 

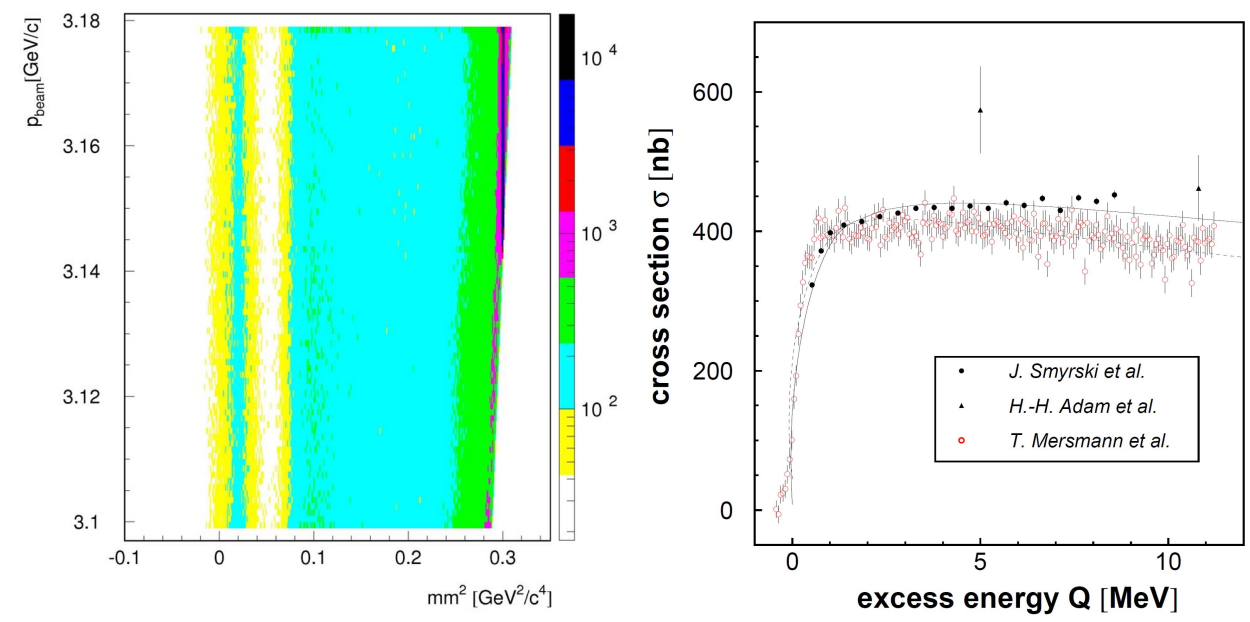

Fig. 3. (Left) Missing mass distribution (horizontal axis) for the $d p \rightarrow{ }^{3} \mathrm{He} X$ reaction as a function of the deuteron beam momentum (vertical axis). The momentum of the deuteron was varied continuously from $3.095 \mathrm{GeV} / c$ to $3.180 \mathrm{GeV} / c$, crossing the threshold for the $d p \rightarrow{ }^{3} \mathrm{He} \eta$ reaction at $3.141 \mathrm{GeV} / c$ [100]. The figure is adapted from reference [100]. (Right) Total cross section for $d p \rightarrow{ }^{3} \mathrm{He} \eta$ plotted as a function of the excess energy $Q$. Shown are the measurements performed by the ANKE Collaboration [93] (open circles) and the COSY-11 Group: [92] (full dots) and [91] (triangles). The solid line represents the scattering length fit to the COSY-11 data [92], while the dashed line is the analogous fit to the data set of Refs. [93, 101]. The figure is adapted from reference [68].

amount to about $270 \mathrm{nb}$ for the $d p \rightarrow\left({ }^{3} \mathrm{He} \eta\right)_{\text {bound }} \rightarrow p p p \pi^{-}$reaction [68], about $70 \mathrm{nb}$ for the $p d \rightarrow\left({ }^{3} \mathrm{He} \eta\right)_{\text {bound }} \rightarrow{ }^{3} \mathrm{He} \pi^{0}$ reaction [68], about $25 \mathrm{nb}$ for the $d d \rightarrow\left({ }^{4} \mathrm{He} \eta\right)_{\text {bound }} \rightarrow{ }^{3} \mathrm{He} n \pi^{0}$ reaction [70], and about $6 \mathrm{nb}$ for the $d d \rightarrow\left({ }^{4} \mathrm{He} \eta\right)_{\text {bound }} \rightarrow{ }^{3} \mathrm{He} p \pi^{-}$reaction [70]. As already pointed out in the previous review [64], these experimental upper limits are close to the recently estimated total cross sections at the $\eta$-mesic pole [102, 103]: $80 \mathrm{nb}$ for the reaction $p d \rightarrow\left({ }^{3} \mathrm{He} \eta\right)_{\text {bound }} \rightarrow X p \pi^{-}$[102] and $4.5 \mathrm{nb}$ [103] to $30 \mathrm{nb}$ [102] for the $d d \rightarrow\left({ }^{4} \mathrm{He} \eta\right)_{\text {bound }} \rightarrow X p \pi^{-}$reaction. The limits are set for the states with the width of few tens of MeV (up to $50 \mathrm{MeV}$ [70]), and do not exclude the much broader state which could be due to the formation of the $N^{*}$ He nuclei, as discussed recently in reference [104]. However, despite the rapid increase of theoretical efforts in recent years, there are still no modelindependent calculations which would really help to plan experiments by judging which out of the ${ }^{4} \mathrm{He} \eta$ and ${ }^{3} \mathrm{He} \eta$ systems is more likely to form a bound state. There are only indirect indications and claims in favor of the ${ }^{3}$ He $\eta$ system, which may be summarized as follows: 
A. The extremely steep rise of the total cross section for the $p d \rightarrow{ }^{3} \mathrm{He} \eta$ reaction (much steeper than in the case of the $d d \rightarrow{ }^{4} \mathrm{He} \eta$ ) in the very close-to-threshold region followed by a plateau may originate from a pole of the $\eta^{3} \mathrm{He} \rightarrow \eta^{3} \mathrm{He}$ scattering amplitude in the complex excess energy plane [92, 93, 101];

B. A steep increase of the total cross section for ${ }^{3} \mathrm{He} \eta$ photoproduction at threshold via the $\gamma^{3} \mathrm{He} \rightarrow \eta^{3} \mathrm{He}$ reaction [74] shows that the rise of the cross section above threshold is independent of the initial channel and can, therefore, be assigned to the ${ }^{3} \mathrm{He} \eta$ interaction;

C. The recent determination of the energy dependence of the tensor analysing power $t_{20}$ by the ANKE Collaboration confirmed that the S-wave production amplitude in the $p d \rightarrow{ }^{3} \mathrm{He} \eta$ reaction is fairly energyindependent [105], again indicating that the steep threshold enhancement is due to the ${ }^{3} \mathrm{He} \eta$ interaction;

D. The asymmetry in the angular distribution of the $\eta$-meson emission $[92,93]$ indicates strong changes of the phase of the s-wave production amplitude with energy, as expected from the occurrence of the bound or virtual $\eta^{3}$ He state [101];

E. The evolution with energy of the angular dependence of $\gamma^{3} \mathrm{He} \rightarrow$ $\eta^{3} \mathrm{He}$ [74] is "similar to that of the $p d \rightarrow{ }^{3} \mathrm{He} \eta$ reaction which indicates changing of s-wave amplitude associated with the $\eta^{3} \mathrm{He}$ " [102];

F. An often stated argument that the extracted $\eta-N$ scattering length is too low for the $\eta$-helium binding is weakened in view of new theoretical results. Estimates of sub-threshold amplitudes are model-dependent and recently Gal et al. has concluded that: "Calculations of $\eta$-nuclear bound states show, in particular, that the $\eta-N$ scattering length is not a useful indicator of whether or not $\eta$ meson binds in nuclei" [106]. Moreover, differences in the value of $\eta-N$ scattering lengths obtained in different analyses are, at least to some extent, explained by the recent observation that the flavor-singlet component induces greater binding than the flavor-octet one. The $\eta-\eta^{\prime}$ mixing, which is neglected in many of the former analyses, may increase the $\eta$-nucleon scattering length relative to the pure octet $\eta$ by a factor of about $2[96,98]$. The importance of the $\eta-\eta^{\prime}$ mixing is also stressed in reference [95].

Motivated by these indications, the WASA-at-COSY Collaboration has performed a high statistics measurement [80] of the $p d$ reactions in the vicinity of the $\eta$-meson production threshold. The data collected in 2014 [80] should allow to investigate eight reactions channels: 
A. Reactions:

$$
\begin{aligned}
& p d \rightarrow\left({ }^{3} \mathrm{He} \eta\right)_{\text {bound }} \rightarrow p p p \pi^{-}, \\
& p d \rightarrow\left({ }^{3} \mathrm{He} \eta\right)_{\text {bound }} \rightarrow p p n \pi^{0}, \\
& p d \rightarrow\left({ }^{3} \mathrm{He} \eta\right)_{\text {bound }} \rightarrow d p \pi^{0}, \\
& p d \rightarrow\left({ }^{3} \mathrm{He} \eta\right)_{\text {bound }} \rightarrow d n \pi^{+}
\end{aligned}
$$

for the test of the hypothesis of absorption of the $\eta$ meson and excitation of one of the nucleons to an $N^{*}(1535)$ resonance, which subsequently decays into an $N-\pi$ pair;

B. Reactions:

$$
\begin{aligned}
& p d \rightarrow\left({ }^{3} \mathrm{He} \eta\right)_{\text {bound }} \rightarrow{ }^{3} \mathrm{He} 2 \gamma \text { and } \\
& p d \rightarrow\left({ }^{3} \mathrm{He} \eta\right)_{\text {bound }} \rightarrow{ }^{3} \mathrm{He} 6 \gamma
\end{aligned}
$$

for the test of the hypothesis of the decay of the $\eta$ meson while "orbiting" around a nucleus [102];

C. Reactions:

$p d \rightarrow\left({ }^{3} \mathrm{He} \eta\right)_{\text {bound }} \rightarrow p p n$,

$p d \rightarrow\left({ }^{3} \mathrm{He} \eta\right)_{\text {bound }} \rightarrow p d$

for the test of non- $N \pi$ decays of the mesic helium pointed out by Wycech [103] and Wilkin [102]. Such processes could be due to the absorption of the $\eta$ meson via $e . g . \eta d \rightarrow p n$ reaction. However, in the first approximation, the two-nucleon absorption constitutes at most $5 \%$ of the total decay rate [102], and in addition, these channels are buried in a large background.

The decay of the $\eta$ meson when it is still orbiting around the nucleus seems to be promising experimentally due to the very low background. Gal et al. pointed out, as a model-independent feature, that "in-medium subthreshold amplitudes encountered in eta-nuclear bound-state calculations are substantially weaker both in their real part as well as in their imaginary part than the $\eta-N$ scattering length" [106]. Decreasing of the imaginary part in the nuclear environment was also observed by Niskanen [107]. This decreases the rate for the process through the $N^{*}$ and its decay into $N-\pi$ but it increases the rate for the decay of the $\eta$ when still orbiting around the nucleus. As a very rough approximation, we may estimate the cross sections for the processes: $p d \rightarrow\left({ }^{3} \mathrm{He} \eta\right)_{\text {bound }} \rightarrow{ }^{3} \mathrm{He} 2 \gamma$, and $p d \rightarrow\left({ }^{3} \mathrm{He} \eta\right)_{\text {bound }} \rightarrow{ }^{3} \mathrm{He} 6 \gamma$ to be about $0.4 \mathrm{nb}$. This value can be estimated taking into account that the total width of the $\eta$ meson is about $1.3 \mathrm{keV}$, the width of the $\left({ }^{3} \mathrm{He} \eta\right)$ is less than about $500 \mathrm{keV}$, and the $2 \gamma$ and $6 \gamma$ branching ratios amount to about 39\% and 33\%, respectively [102]. 
It is worth to point out that the WASA detector at COSY gave unique possibilities to conduct the measurement of the above listed reactions with the continuous change of the beam momentum and for five of them (without neutron in the final state), an exclusive measurement of all ejectiles was possible. The left panel of Fig. 3 shows an exemplary spectrum [100] from COSY, illustrating the power of the possibility of continuous change of the beam momentum during the experimental run. This technique permits to decrease the systematic errors of relative normalization with respect to the measurements carried out for each momentum value separately.

The author acknowledges very useful comments and corrections of the manuscript by M. Baskhanov, S. Bass, H. Clement, N. Kelkar, W. Krzemień and M. Nowakowski, and the partial support of the work by the Polish National Science Centre through grants Nos. 2011/03/B/ST2/01847 and 2011/01/B/ST2/00431, and by the Foundation for Polish Science (MPD programme).

\section{REFERENCES}

[1] M. Gell-Mann, Phys. Lett. 8, 214 (1964).

[2] R.L. Jaffe, Phys. Rev. D 15, 281 (1977).

[3] S.L. Olsen, Front. Phys. 10, 101401 (2015).

[4] L. Maiani et al., Phys. Rev. Lett. 93, 212002 (2004).

[5] F.-K. Guo et al., Phys. Rev. D 88, 074506 (2013).

[6] C.A. Meyer, E.S. Swanson, Prog. Part. Nucl. Phys. 82, 21 (2015).

[7] S.D. Bass, E. Marco, Phys. Rev. D 65, 057503 (2002).

[8] S.K. Choi et al., Phys. Rev. Lett. 91, 262001 (2003).

[9] B. Aubert et al., Phys. Rev. D 71, 071103 (2005).

[10] R. Aaij et al., Phys. Rev. Lett. 110, 222001 (2013).

[11] P. del Amo Sanchez et al., Phys. Rev. D 82, 011101 (2010).

[12] R. Aaij et al., Nucl. Phys. B 886, 665 (2014).

[13] D. Acosta et al., Phys. Rev. Lett. 93, 072001 (2004).

[14] A. Abulencia et al., Phys. Rev. Lett. 98, 132002 (2007).

[15] V.M. Abazov et al., Phys. Rev. Lett. 93, 162002 (2004).

[16] R. Aaij et al., Eur. Phys. J. C 72, 1972 (2012).

[17] S. Chatrchyan et al., J. High Energy Phys. 1304, 154 (2013).

[18] M. Ablikim et al., Phys. Rev. Lett. 112, 092001 (2014). 
[19] V. Bhardwaj et al., Phys. Rev. Lett. 107, 091803 (2011).

[20] N.A. Tornqvist, Phys. Lett. B 590, 209 (2004).

[21] L. Maiani et al., Phys. Rev. D 71, 014028 (2005).

[22] C. Hanhart et al., Eur. Phys. J. A 47, 101 (2011).

[23] R. Aaij et al., Phys. Rev. Lett. 115, 072001 (2015).

[24] M. Karliner, J. Rosner, Phys. Rev. Lett. 115, 122001 (2015).

[25] M. Bashkanov, private communication, 2015.

[26] J.M.M. Hall et al., Phys. Rev. Lett. 114, 132002 (2015).

[27] T. Inoue et al., Phys. Rev. C 65, 035204 (2002).

[28] B.S. Zou et al., Phys. Rev. C 87, 035206 (2013).

[29] M. Praszalowicz, Phys. Lett. B 575, 234 (2003).

[30] N.G. Kelkar et al., J. Phys. G 29, 1001 (2003).

[31] T. Nakano et al., Phys. Rev. Lett. 91, 012002 (2003).

[32] V.V. Barmin et al., Phys. Atom. Nucl. 66, 1715 (2003).

[33] M. Battaglieri et al., Phys. Rev. Lett. 96, 042001 (2006).

[34] M. Moritsu et al., Phys. Rev. C 90, 035205 (2014).

[35] K. Shirotori et al., Phys. Rev. Lett. 109, 132002 (2012).

[36] M. Naruki, Acta Phys. Pol. B 47, (2016), in print.

[37] V.V. Barmini et al., Phys. Rev. C 89, 045204 (2014).

[38] P. Adlarson et al., Phys. Rev. Lett. 106, 242302 (2011).

[39] P. Adlarson et al., Phys. Rev. Lett. 112, 202301 (2014).

[40] F.J. Dyson, N.-H. Xuong, Phys. Rev. Let. 13, 815 (1964).

[41] B.S. Neganov, L.B. Parfenov, Zh. Eksp. Teor. Fiz. 34, 767 (1958);

M.G. Meshcheriakov, B.S. Neganov, Dokl. Akad. Nauk SSSR 100, 677 (1955).

[42] M. Bashkanov et al., Phys. Rev. Lett. 102, 052301 (2009).

[43] P. Adlarson et al., Phys. Lett. B 721, 229 (2013).

[44] M. Bashkanov, Talk at the Jagiellonian Symposium on Fundamental and Applied Subatomic Physics, Cracow 2015; Acta Phys. Pol. B 47, (2016) in print.

[45] G. Fäldt, C. Wilkin, Phys. Lett. B 701, 619 (2011).

[46] M. Albaladejo, E. Oset, Phys. Rev. C 88, 014006 (2013).

[47] P. Adlarson et al., Phys. Lett. B 743, 325 (2015).

[48] P. Adlarson et al., Phys. Rev. C 88, 055208 (2013).

[49] P. Adlarson et al., Phys. Rev. C 90, 035204 (2014).

[50] P. Adlarson et al., Phys Rev. C 91, 015201 (2015).

[51] M. Bashkanov et al., Phys. Lett. B 637, 223 (2006).

[52] P. Adlarson et al., Phys. Rev. C 86, 032201(R) (2012). 
[53] J. Ball et al., Nucl. Phys. A 559, 489 (1993); A. de Lesquen et al., Eur. Phys. J. C 11, 69 (1999); Y. Makdisi et al., Phys. Rev. Lett. 45, 1529 (1980); C.R. Newsom et al., Phys. Rev. C 39, 965 (1989); J. Arnold et al., Eur. Phys. J. C 17, 67 (2000); J. Ball et al., Nucl. Phys. B 286, 635 (1987); M.W. McNaughton et al., Phys. Rev. C 48, 256 (1993); 53, 1092 (1996); G. Glass et al., Phys. Rev. C 47, 1369 (1993).

[54] M. Bashkaov, H. Clement, T. Skorotko, Eur. Phys. J. A 51, 87 (2015).

[55] A. Pricking, M. Bashkanov, H. Clement, arXiv:1310.5532 [nucl-ex].

[56] M. Bashkanov, H. Clement, D.P. Watts, arXiv:1508.07163 [nucl-ex].

[57] F. Huang, J. Ping, F. Wang, Phys. Rev. C 89, 034001 (2014).

[58] A. Gal, H. Garcilazo, Phys. Rev. Lett. 111, 172301 (2013).

[59] A. Gal, H. Garcilazo, Nucl. Phys. A 928, 73 (2014).

[60] A. Gal, Acta Phys. Pol. B 47, (2016), in print [arXiv:1511.06605 [nucl-th]].

[61] H. Garcilazo, A. Gal, Nucl. Phys. A 897, 167 (2013).

[62] A. Gal et al., Phys. Rev. D 90, 014019 (2014).

[63] S. Bass, P. Moskal, arXiv:1510.06202 [hep-ph]; Acta Phys. Pol. B 47, (2016), in print; C. Wilkin, Acta Phys. Pol. B 47, (2016), in print; Q. Haider, L.C. Liu, Int. J. Mod. Phys. E 24, 1530009 (2015); H. Machner, J. Phys. G 42, 043001 (2015); N.G. Kelkar, Acta Phys. Pol. B 46, 113 (2015); B. Krusche, C. Wilkin, Prog. Part. Nucl. Phys. 80, 43 (2014); N.G. Kelkar et al., Rep. Prog. Phys. 76, 066301 (2013); E. Friedman, A. Gal, J. Mares, Phys. Lett. B 725, 334 (2013).

[64] P. Moskal, Few Body Syst. 55, 667 (2014).

[65] Q. Haider, L.C. Liu, Phys. Lett. B 172, 257 (1986).

[66] R.E. Chrien et al., Phys. Rev. Lett. 60, 2595 (1988).

[67] A. Budzanowski et al., Phys. Rev. C 79, 012201 (2009).

[68] P. Moskal, J. Smyrski, Acta Phys. Pol. B 41, 2281 (2010).

[69] P. Adlarson et al., Phys. Rev. C 87, 035204 (2013).

[70] M. Skurzok et al., Acta Phys. Pol. B 47, (2016), in print.

[71] W. Krzemien et al., Acta Phys. Pol. B 46, 757 (2015).

[72] M. Skurzok et al., Prog. Part. Nucl. Phys. 67, 445 (2012).

[73] S.V. Afanasiev et al., Phys. Part. Nucl. Lett. 8, 1073 (2011).

[74] F. Pheron et al., Phys. Lett. B 709, 21 (2012).

[75] V.A. Baskov et al., PoS Baldin-ISHEPP-XXI, 102 (2012).

[76] K. Itahashi et al., Prog. Theor. Phys. 128, 601 (2012).

[77] V. Metag et al., approved by ELSA/03-2012-BGO-OD.

[78] N. Muramtsu, arXiv:1307.6411 [physics.ins-det].

[79] B. Krusche et al., J. Phys.: Conf. Ser. 349, 012003 (2012).

[80] P. Moskal, W. Krzemien, M. Skurzok, COSY proposal No. 186.3 (2014). 
[81] H. Fujioka et al., Acta Phys. Pol. B 41, 2261 (2010).

[82] A.V. Afanasiev et al., Nucl. Phys. Proc. Suppl. 245, 173 (2013).

[83] P. Moskal et al., Phys. Lett. B 482, 356 (2000).

[84] P. Moskal et al., Phys. Rev. C 69, 025203 (2004).

[85] E. Czerwinski et al., Phys. Rev. Lett. 113, 062004 (2014).

[86] P. Moskal et al., Prog. Part. Nucl. Phys. 49, 1 (2002).

[87] B. Krusche et al., Eur. Phys. J. A 22, 277 (2004); M. Röbig-Landau et al., Phys. Lett. B 373, 45 (1996); T. Mertens et al., Eur. Phys. J. A 38, 195 (2008).

[88] M. Nanova et al., Phys. Lett. B 710, 600 (2012).

[89] C. Wilkin, Acta Phys. Pol. B 47, (2016) in print.

[90] J. Berger et al., Phys. Rev. Lett. 61, 919 (1988); B. Mayer et al., Phys. Rev. C 53, 2068 (1996); M. Betigeri et al., Phys. Lett. B 472, 267 (2000); J. Smyrski et al., Acta Phys. Slov. 56, 213 (2006).

[91] H.H. Adam et al., Phys. Rev. C 75, 014004 (2007).

[92] J. Smyrski et al., Phys. Lett. B 649, 258 (2007).

[93] T. Mersmann et al., Phys. Rev. Lett. 98, 242301 (2007).

[94] R. Frascaria et al., Phys. Rev. C 50, 537 (1994); N. Willis et al., Phys. Lett. B 406, 14 (1997); A. Wronska et al., Eur. Phys. J. A 26, 421 (2005);

A. Budzanowski et al., Nucl. Phys. A 821, 193 (2009).

[95] S. Hirenzaki, H. Nagahiro, Acta Phys. Pol. B 45, 619 (2014).

[96] S.D. Bass, A.W. Thomas, Phys. Lett. B 634, 368 (2006).

[97] S. Hirenzaki et al., Acta Phys. Pol. B 41, 2211 (2010).

[98] S.D. Bass, A.W. Thomas, Acta Phys. Pol. B 45, 627 (2014).

[99] M. Nanova et al., Phys. Lett. B 727, 417 (2013).

[100] J. Smyrski et al., Nucl. Phys. A 790, 438 (2007).

[101] C. Wilkin, Phys. Lett. B 654, 92 (2007).

[102] C. Wilkin, Acta Phys. Pol. B 45, 603 (2014).

[103] S. Wycech, W. Krzemien, Acta Phys. Pol. B 45, 745 (2014).

[104] N. Kelkar et al., Acta Phys. Pol. B 47, (2016), in print.

[105] M. Papenbrock et al., Phys. Lett. B 734, 333 (2014).

[106] A. Gal et al., Acta Phys. Pol. B 45, 673 (2014).

[107] J. Niskanen, arXiv:1502.03278 [nucl-th]. 